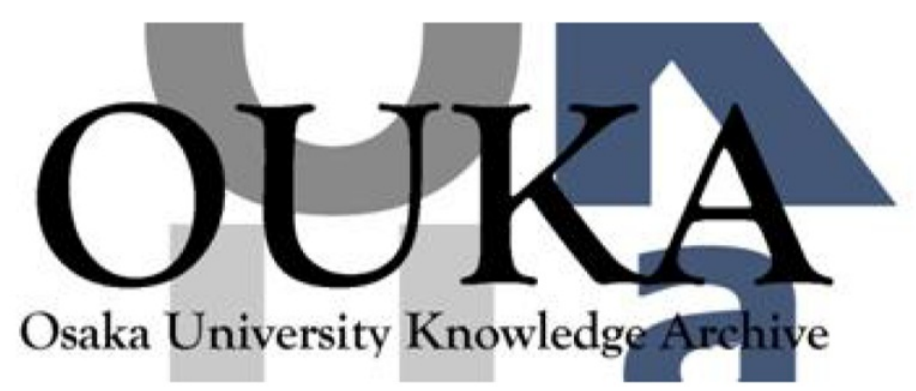

\begin{tabular}{|c|l|}
\hline Title & $\begin{array}{l}\text { A modular approach to large-signal modeling of } \\
\text { an interconnected AC/MTDC system }\end{array}$ \\
\hline Author(s) & $\begin{array}{l}\text { Susuki, Yoshihiko; Kawamoto, Naoki; Ohashi, } \\
\text { Yusuke et al. }\end{array}$ \\
\hline Citation & $\begin{array}{l}\text { IEEE PES Innovative Smart Grid Technologies } \\
\text { Conference Europe. p.945-p.949 }\end{array}$ \\
\hline Issue Date & $2020-10-26$ \\
\hline oaire:version & AM \\
\hline URL & $\begin{array}{l}\text { https://hdl. handle. net/11094/78283 } \\
\text { O 2020 IEEE. Personal use of this material is } \\
\text { permitted. Permission from IEEE must be } \\
\text { obtained for all other uses, in any current or } \\
\text { future media, including reprint ing/republishing } \\
\text { this material for advertising or promotional } \\
\text { purposes, creating new collect ive works, for } \\
\text { resale or redistribution to servers or lists, } \\
\text { or reuse of any copyrighted component of this } \\
\text { work in other works. }\end{array}$ \\
\hline rights \\
\hline Note & \begin{tabular}{l} 
\\
\hline
\end{tabular} \\
\hline
\end{tabular}

Osaka University Knowledge Archive : OUKA

https://ir. Library. osaka-u. ac. jp/

Osaka University 


\section{A Modular Approach to Large-Signal Modeling of an Interconnected AC/MTDC System}

\author{
Yoshihiko Susuki, Naoki Kawamoto \\ Yusuke Ohashi, Atsushi Ishigame \\ Osaka Prefecture University \\ Sakai, Japan \\ susuki@eis.osakafu-u.ac.jp
}

\author{
Tsuyoshi Funaki \\ Osaka University \\ Suita, Japan
}

\author{
Salvatore D'Arco \\ SINTEF Energy Research \\ Trondheim, Norway
}

\begin{abstract}
The so-called modular modeling of interconnected AC/MTDC (Multi-Terminal DC) systems aids their synthesis and analysis by decomposing it into structural modules (e.g. voltage-source converter, synchronous generator, AC line, DC line) and by exploring the patterns of interconnection that yield the overall performance of a system. In this paper, we introduce our idea on the modular modeling of large-signal dynamics for an interconnected AC/MTDC system. Effectiveness of the idea is shown with numerical simulations for a relatively simple configuration of three-terminal DC grid.

Index Terms-Multi-terminal direct current; Large-signal modeling; Numerical simulation; Power system performance assessment
\end{abstract}

\section{INTRODUCTION}

Multi-Terminal DC (MTDC) grids based on Voltage-Source Converters (VSCs) have been widely studied: see, e.g., [1] By virtue of the control capability of VSCs, the MTDC grids are expected to provide flexibility in the operation of conventional AC grids. Most of practical MTDC grids are now in the planning phase, therefore it is relevant to rely on the so-called model-based approach to synthesis and analysis of static/dynamic performances for interconnected systems of AC/MTDC grids. For this, it is required to construct a generic mathematical model capturing the physical characteristics of a target MTDC grid and to integrate the model with conventional models of the AC grids for exploring various patterns of interconnection of our interest. No general framework of the integration applicable to a wide class of the AC/MTDC interconnection has been reported yet to the best of the authors' knowledge.

The so-called modular modeling of interconnected AC/MTDC systems aids their synthesis and analysis by decomposing it into structural modules (e.g. VSC, synchronous generator, AC line, DC line) and by exploring the patterns of interconnection that yield the overall performance of a system such as economic efficiency (optimality) and physical stability. In this paper, we introduce our idea on the modular modeling of large-signal dynamics for a relatively-simple model of interconnected AC/MTDC systems. The large-signal dynamics and associated stability have a long of history of research on interconnected AC/DC systems: see, e.g., [2]-[4]. The modular modeling is motivated by not only software development for generic use [5] but also system-theoretic perspective [6], [7]. For the modular modeling, in this paper we reformulate existing dynamic models of a synchronous generator and a DC line (phasorbased for AC and instantaneous variable-based for DC), called modules, so that they can be utilized in the modular modeling. In addition to the reformulation, by virtue of the switching function to represent the ON/OFF operation of IGBT valves,

This work is supported in part by JST SICORP Grant Number JPMJSC17C2, Japan. which is utilized in [4] for current-source converters, we derive a basic mathematical model of VSC that represents its power conversion with a simple control mechanism, which is expected to be minimal for the large-signal studies. The power-based approach makes it possible to explore the patterns of interconnection of various components in AC and MTDC grids, in order to assess the overall largesignal dynamics and stability. Effectiveness of the modular modeling is examined with numerical simulations for multiple configurations of the MTDC network. A preliminary version of this paper is the non-reviewed technical report [8] inside Japan that includes the list of parameter values used in this paper.

There are several works related to the contents of this paper. In [9], a generic model for VSC-based MTDC consisting of converter and its controllers is proposed. In [10], a combination of the linearized classical swing equation and DC circuit equations with PI-type converter controller is used for design of distributed frequency controller. In [11], the sixthorder model for generator dynamics with models of MMC (Modular Multilevel Converter) and DC cable are introduced for assessment of small-signal stability. In the present paper, we derive a relatively simple model of VSC by a common formula of power conversion in [9] and thus a set of nonlinear differential and algebraic equations for the large-signal dynamics and stability.

\section{System Model}

Figure 1 shows a single-line diagram of the interconnected system of AC/MTDC grids for which we demonstrate the modular modeling. The system model includes the two AC grids (left and right sides of the figure) asynchronously operated onto one MTDC grid like [11]. The left AC grid has one synchronous generator and two VSCs, called VSC\#1 and VSC\#2, and the grid's dynamics and stability are of basic interest for the large-signal modeling. The right $\mathrm{AC}$ grid has one VSC, called VSC\#3, and no generator; thus the associated dynamics are not considered. Each of the AC grids includes the infinite bus as a synchronously-rotating reference axis. In the MTDC network, we suppose from wide use in practical systems that it is of bipolar-type with a return conductor [12], as shown in Section III-C.

\section{MODELING FRAMEWORK:}

\section{MODUle MODELS AND THEIR INTERCONNECTION}

This section describes the modular approach to largesignal modeling of the interconnected system in Figure 1. Specifically, we reinterpret the standard models of dynamics of synchronous generator, AC and DC line as modules, and we derive a basic model of VSC as an essential element connecting the AC and MTDC grids. Here, we make the following three assumptions: 


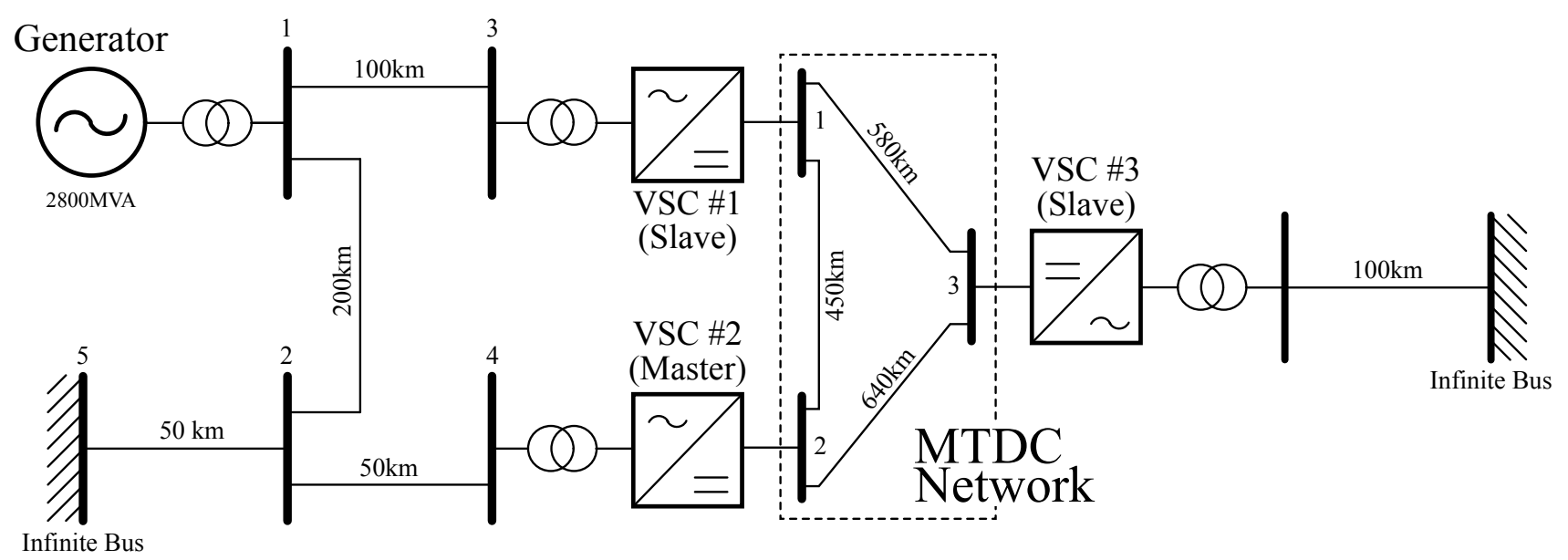

Fig. 1. Single-line diagram of the interconnected system of AC/MTDC grids for which we demonstrate the modular modeling in this paper

A1 For the AC grids, we focus on the timescale of electromechanical dynamics (normally, 0 to 10 seconds) that is essential in the large-signal studies. Any electrical transient in AC lines and transformers are thus negligible. In addition, three-phase voltages are balanced in steady and transient states.

A2 For the bipolar-type MTDC network, voltages and currents in positive and negative-side circuits are balanced in steady and transient states.

A3 For the three VSCs, no power loss exists, and switching operations of IGBT-based valves are ideal.

Assumption A1 is again standard in the large-signal studies. Assumptions A2 and A3 are used for characterizing electromechanical dynamics of synchronous generators operating onto both AC and DC grids [4], [13]. All the variables and parameters are in per-unit systems for the AC and MTDC grids: see [13] in details.

\section{A. Synchronous Generator}

We represent the electromechanical dynamics of the synchronous generator in Figure 1 using the so-called one-axis model with exciter [14]. For the generator, we denote $\delta$ by the angular position of rotor with respect to the infinite bus. The non-dimensional deviation of rotor speed relative to a nominal angular frequency is denoted by $\omega$, and the non-dimensional voltage behind transient reactance by $e_{q}^{\prime}$. The model is then given as

$$
\left.\begin{array}{rl}
\frac{\mathrm{d} \delta}{\mathrm{d} t} & =\omega \\
2 H \frac{\mathrm{d} \omega}{\mathrm{d} t} & =-D \omega+P_{\mathrm{m}}-p \\
T_{d 0}^{\prime} \frac{\mathrm{d} e_{q}^{\prime}}{\mathrm{d} t} & =\frac{X_{d}-X_{d}^{\prime}}{X_{d}^{\prime}} v_{1} \cos \left(\delta-\theta_{1}\right)+E_{f d}-\frac{X_{d}}{X_{d}^{\prime}} e_{q}^{\prime}
\end{array}\right\}
$$

where $H, D, T_{d 0}^{\prime}, X_{d}\left(X_{q}\right), X_{d}^{\prime}$, and $E_{f d}$ are parameters of the generator. The mechanical input power $P_{\mathrm{m}}$ is constant under Assumption A1. The electric output of active power $p$ and associated reactive power $q$ are given by

$$
\left.\begin{array}{rl}
p= & \frac{e_{q}^{\prime} v_{1}}{X_{d}^{\prime}} \sin \left(\delta-\theta_{1}\right)+\frac{v_{1}^{2}}{2} \frac{X_{d}^{\prime}-X_{q}}{X_{d}^{\prime} X_{q}} \sin 2\left(\delta-\theta_{1}\right) \\
q= & \frac{e_{q}^{\prime} v_{1}}{X_{d}^{\prime}} \cos \left(\delta-\theta_{1}\right)-\frac{v_{1}^{2}}{2} \frac{X_{d}^{\prime}+X_{q}}{X_{d}^{\prime} X_{q}} \\
& +\frac{v_{1}^{2}}{2} \frac{X_{d}^{\prime}-X_{q}}{X_{d}^{\prime} X_{q}} \cos 2\left(\delta-\theta_{1}\right)
\end{array}\right\} .
$$

The variables $\theta_{1}$ and $v_{1}$ are the phase and amplitude of voltage phasor at AC bus \#1 onto which the generator is operated. We state that the 4-tuple $\left(p, q, \theta_{1}, v_{1}\right)$ is the set of variables sharing to express interconnections [6], that is, to link the generator's model ("module") with the AC lines below, which is conventional in power system studies.

\section{B. AC Lines}

From Assumption A1, we simply represent AC lines in Figure 1 using the power-flow model. For two AC buses in the left grid, which are labeled by integers $i$ and $j$ from $\{1, \ldots, 5\}$ and connected by $\mathrm{AC}$ lines, we have

$$
\left.\begin{array}{rl}
p_{i}= & v_{i} v_{j}\left\{G_{i j} \cos \left(\theta_{i}-\theta_{j}\right)+B_{i j} \sin \left(\theta_{i}-\theta_{j}\right)\right\} \\
& +v_{i}^{2} G_{i i} \\
q_{i}= & v_{i} v_{j}\left\{G_{i j} \sin \left(\theta_{i}-\theta_{j}\right)-B_{i j} \cos \left(\theta_{i}-\theta_{j}\right)\right\} \\
& -v_{i}^{2} B_{i i}
\end{array}\right\}
$$

where $\theta_{i}$ and $v_{i}$ are the phase and amplitude of voltage phasor at $\mathrm{AC}$ bus $\# i$, and $G_{i j}$ and $B_{i j}$ are parameters of the $\mathrm{AC}$ lines. The active power $p_{i}$ and reactive power $q_{i}$ are terms determined by a generator, load, and VSC connected to AC bus \#i. The 4-tuple $\left(p, q, \theta_{i}, v_{i}\right)$ is hence the set of variables sharing to express interconnections, namely, to link the ACline module with another.

\section{DC Lines}

A $\pi$-type equivalent circuit of bipolar DC lines is shown in Figure 2 , where $R_{\mathrm{dc} \text { line }}, L_{\mathrm{dc} \text { line }}$, and $C_{\mathrm{dc} \text { line }}$ are constant parameters. The ports on the left and right sides of the figure correspond to DC buses for which we use the integer labels $i$ and $j$. Under Assumption A2, in order to consider a possible fault in the DC network and associated electrical transient, we 


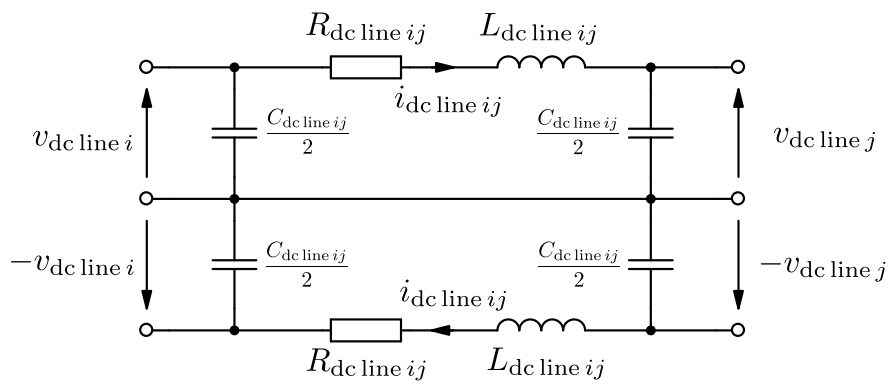

Fig. 2. $\pi$-type equivalent circuit of bipolar-type (symmetrical) DC lines

use the governing equations for the DC current $i_{\mathrm{dc} \text { line } i j}$ and the DC voltage $v_{\mathrm{dc}}$ line $i$ as

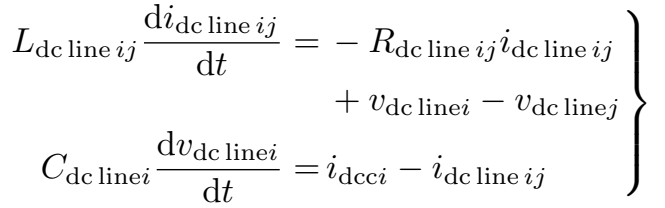

where $i_{\mathrm{dcc} i}$ stands for the DC current flowing into DC bus $\# i$ from an AC grid through VSC and will be a key variable below. This shows that the tuple $\left(v_{\mathrm{dcline} i}, i_{\mathrm{dcc} i}\right)$ is the set of variables sharing to express interconnections, that is, to link the DC-line module with another. It is noted that if DC bus $\# i$ has no VSC, then the associated $i_{\mathrm{dcc} i}$ is negligible.

\section{Voltage-Source Converters (I)-Physical}

Above, we introduced the modules for $\mathrm{AC}$ generator and AC lines with the variables $(p, q, \theta, v)$ and the module for DC lines with $\left(v_{\mathrm{dc}}, i_{\mathrm{dc}}\right)$. To share their variables at a VSC, we now derive a basic model of VSC based on the conservation law of power.

First, we consider the relation between voltages on $\mathrm{AC}$ and DC sides at a VSC. Let us denote by $e_{d}$ and $e_{q}$ the $d$ and $q$-axis voltages at the AC side $^{1}$, and by $v_{\mathrm{dc}}$ the positiveside voltage at the DC side which magnitude is equal to the negative-side voltage under Assumption A2. The $v_{\text {dc }}$ can be identified as $i_{\mathrm{dc} \text { line } i}$ in Figure 2 . Needless to say, $e_{d}$ and $e_{q}$ are functions of the phase $\theta$ and amplitude $v$ of voltage phasor at the corresponding $\mathrm{AC}$ bus. By focusing on the harmonic component of voltage waveform at the AC side through the switching function, the following approximate relation among $e_{d}, e_{q}$, and $v_{\mathrm{dc}}$ is derived in [8]:

$$
e_{d}=2 K_{V} v_{\mathrm{dc}} u_{d}, \quad e_{q}=2 K_{V} v_{\mathrm{dc}} u_{q},
$$

with the two control variables

$$
u_{d}:=\frac{2}{\pi} \sin \frac{\alpha}{2} \sin \theta, \quad u_{q}:=-\frac{2}{\pi} \sin \frac{\alpha}{2} \cos \theta,
$$

or

$$
\left.\begin{array}{l}
v:=\sqrt{e_{d}^{2}+e_{q}^{2}}=2 K_{V} v_{\mathrm{dc}} u \\
u:=\sqrt{u_{d}^{2}+u_{q}^{2}}=\frac{2}{\pi} \sin \frac{\alpha}{2}
\end{array}\right\}
$$

where $\alpha \in[0, \pi]$ stands for the conduction duration of IGBT valves of VSC, and $\theta$ for the phase of voltage phasor at the AC side. The constant $K_{V}$ in (5) and (7) is determined by the choice of base quantities in the per-unit systems of $\mathrm{AC}$ and MTDC grids: see [13] in details.

\footnotetext{
${ }^{1}$ Under Assumption A1, there is no zero-axis voltage.
}

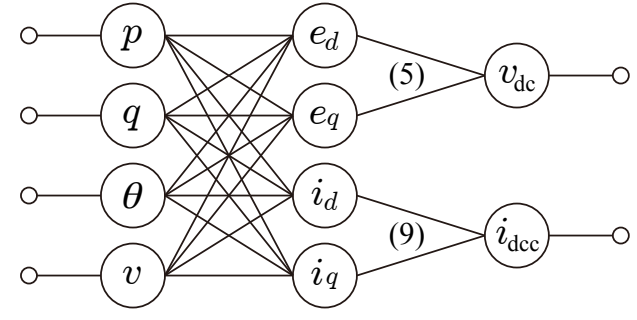

Fig. 3. Graphical representation of sharing the variables at Voltage-Source Converter (VSC)

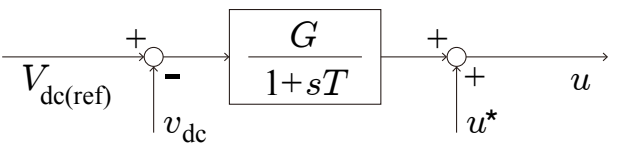

(a) Master Control

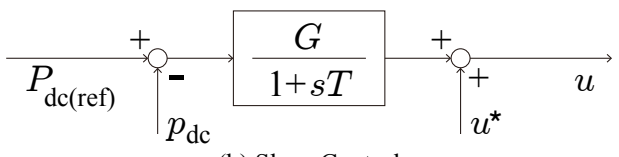

(b) Slave Control

Fig. 4. Block diagrams of Master-Slave Control for Voltage-Source Converter (VSC)

Second, we consider the relation between currents at the VSC. From Assumption A3, by denoting as $i_{d}$ and $i_{q}$ the $d$ and $q$-axis currents at the $\mathrm{AC}$ side, the conservation law of active power in the per-unit systems yields

$$
p=e_{d} i_{d}+e_{q} i_{q}=v_{\mathrm{dc}} i_{\mathrm{dcc}}
$$

where $i_{\mathrm{dcc}}$ appears in (4). Thus, from (5), the following relation among $i_{d}, i_{q}$, and $i_{\mathrm{dcc}}$ is derived in [8]:

$$
\sqrt{3} K_{I}\left(u_{d} i_{d}+u_{q} i_{q}\right)=i_{\mathrm{dcc}}
$$

where we note $\sqrt{3} K_{I}$ is the constant determined by the perunit systems. The $i_{d}$ and $i_{q}$ are determined with $\left(e_{d}, e_{q}, p\right)$ and $q=e_{q} i_{d}-e_{d} i_{q}$ [15]. Hence, the static VSC model is established as the power conversion (8) and the power-based conversion of variables in Figure 3.

\section{E. Voltage-Source Converters (II)-Control}

Finally, we introduce a control model of VSC. There is a lot of research and development of control systems for VSCs: see, e.g., [12], [16]. It is valuable to explore which type of control mechanism is minimal for explaining the target large-signal dynamics and stability; this is an open question. In this paper, as a preliminary work, we adopt the idea of Master-Slave Control that is investigated in practice [17] and studied in [18]. In this, one VSC, called Master-VSC, aims to regulate a DC voltage while another, called Slave-VSC, to regulate active/reactive power. By assuming that internal control dynamics are sufficiently fast and thus negligible in the timescale of electromechanical dynamics, we consider the Master-Slave Control as shown in Figure 4, where $u$ in (7) is the control variable, $u^{*}$ its steady-state value, and $V_{\mathrm{dc}(\mathrm{ref})}$ (or $P_{\mathrm{dc}(\mathrm{ref})}$ ) the set-point of voltage (or power) regulation at a Master-VSC (or Slave-VSC). The first-order lag element represents the inevitable lag effect of signals in measurement and actuation. Needless to say, there is room for improvement of the modeling in terms of large-signal dynamics and stability. One idea is to incorporate the so-called droop-type control 
(see, e.g., [19]) to the current model, which is in our future research.

\section{F. Model Development by Interconnection}

By connecting the above modules, we are able to develop a nonlinear dynamic model for the interconnected system of AC/MTDC grids. To this end, we need to modify the module models of AC lines and DC lines according to the graph structures of AC and MTDC transmission networks, that is to say, how the module models are connected in the target system.

For simplicity of the presentation, we now focus in Figure 1 on the left AC grid and MTDC grid: the following modification holds for the right $\mathrm{AC}$ grid. Now, following [7], [10], let us represent the AC and MTDC networks using directed graphs $\mathcal{G}_{\mathrm{AC}}=\left(\mathcal{V}_{\mathrm{AC}}, \mathcal{E}_{\mathrm{AC}}\right)$ and $\mathcal{G}_{\mathrm{DC}}=\left(\mathcal{V}_{\mathrm{DC}}, \mathcal{E}_{\mathrm{DC}}\right)$ with the set of $\mathrm{AC}$ (or DC) buses $\mathcal{V}_{\mathrm{AC}}=\{1,2, \ldots, 5\}$ (or $\left.\mathcal{V}_{\mathrm{DC}}=\{1,2,3\}\right)$ and $\mathrm{AC}$ lines $\mathcal{E}_{\mathrm{AC}} \subseteq \mathcal{V}_{\mathrm{AC}} \times \mathcal{V}_{\mathrm{AC}}$ (or DC lines $\mathcal{E}_{\mathrm{DC}} \subseteq \mathcal{V}_{\mathrm{DC}} \times$ $\left.\mathcal{V}_{\mathrm{DC}}\right)$. Regarding software utilization, the graph structures $\mathcal{G}_{\mathrm{AC}}$ and $\mathcal{G}_{\mathrm{DC}}$ can be recursively updated by users (modelers) who analyze interconnected systems of interest. For each update, the modules of AC and DC lines are modified in the following manner.

The following modification of the module of AC lines is well-known in the traditional power-flow analysis. For this, the neighbors of AC bus $\# i$ are denoted by $\mathcal{N}_{\mathrm{AC} i}:=\left\{j \in \mathcal{V}_{\mathrm{AC}}\right.$ : $\left.(i, j) \in \mathcal{E}_{\mathrm{AC}}\right\}$. Then, the power-flow model (3) is modified as follows:

$$
\left.\begin{array}{rl}
p_{i}= & \sum_{j \in \mathcal{N}_{\mathrm{AC} i}} v_{i} v_{j}\left\{G_{i j} \cos \left(\theta_{i}-\theta_{j}\right)+B_{i j} \sin \left(\theta_{i}-\theta_{j}\right)\right\} \\
& +v_{i}^{2} G_{i i} \\
q_{i}= & \sum_{j \in \mathcal{N}_{\mathrm{AC} i}} v_{i} v_{j}\left\{G_{i j} \sin \left(\theta_{i}-\theta_{j}\right)-B_{i j} \cos \left(\theta_{i}-\theta_{j}\right)\right\} \\
& -v_{i}^{2} B_{i i}
\end{array}\right\}
$$

Also, if AC bus \# $i$ is connected to a VSC, then the corresponding voltage amplitude $v_{i}$ is related to the DC-bus voltage with (7).

We next show a similar modification of the module of DC lines. The neighbors of DC bus \#i are denoted by $\mathcal{N}_{\mathrm{DC} i}:=$ $\left\{j \in \mathcal{V}_{\mathrm{DC}}:(i, j) \in \mathcal{E}_{\mathrm{DC}}\right\}$. For DC bus $\# i$, by considering the smoothing capacitance $C_{\mathrm{dc} \text { conv }}$ of VSC, the total capacitance $C_{\mathrm{dc} i}$ is defined as

$$
C_{\mathrm{dc} i}:=C_{\mathrm{dc} \text { conv }}+\sum_{j \in \mathcal{N}_{\mathrm{DC} i}} \frac{C_{\mathrm{dc} \text { line } i j}}{2} .
$$

Thus, the circuit dynamics described by (4) are modified as follows:

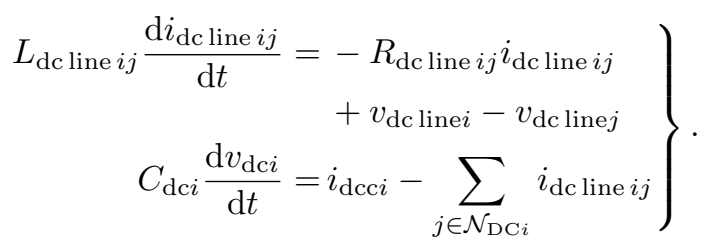

Therefore, we are able to build up the full model of the interconnected AC/MTDC system as a set of differential and algebraic equations described by $(1,12)$ and $(2,8,10)$, with the control mechanism of VSC in Figure 4.

It should be noted that the above modular modeling is explained for the simple system in Figure 1, and that the same modeling is applicable to more complicated systems without any substantial modification.

\section{NUMERICAL SimUlations}

In this section, we use the modular modeling for numerical simulations of large-signal dynamics for the interconnected AC/MTDC system in Figure 1. To show the effectiveness of the modular modeling, transient simulations for multiple configurations of the MTDC network were conducted as described below. The change of the configuration is easily realized in the modular modeling by updating the graph data $\mathcal{G}_{\mathrm{DC}}$. The values of parameters for the AC and MTDC grids are based on standard setting in literature, e.g., [4], [13] and available in our technical report [8]. The control parameters of the three VSCs are $G=-1, T=1 \mathrm{~ms}, V_{\mathrm{dc}(\mathrm{ref})}=1$ for VSC\#2, $P_{\mathrm{dc}(\mathrm{ref})}=0.2$ for VSC\#1, and $P_{\mathrm{dc}(\mathrm{ref})}=-0.3$ for VSC\#3. Unfortunately, there is no space in this paper that shows the complete list of the values of parameters. Numerical solutions of the set of nonlinear differential and algebraic equations were obtained with implicit trapezoidal method with fixed time-step of $1 \mathrm{~ms}$. We supposed that at $t=0 \mathrm{~s}$ the system was at a normal operating condition (that is, a stable equilibrium point of the nonlinear dynamic model), and at $t=0.1 \mathrm{~s}$ a severe contingency occurred in the AC grid. The fault was encoded as a change of the initial condition from the equilibrium point: the value of $\omega$ at $t=0.1 \mathrm{~s}$ was increased by $0.02(1 \mathrm{~Hz})$.

Figure 5 shows the numerical simulations of the modular model for the four different configurations of the MTDC network. The four configurations are (a) all-to-all coupling, which is the same as in Figure 1, denoted by the black lines, (b) the MTDC network without line 1-2 denoted by the red lines, (c) the MTDC network without line 1-3 denoted by the green lines, and (d) the MTDC network without line 2-3 denoted by the blue lines. In this figure, the transient dynamics of the left AC grid are almost the same for the different configurations. This results from the setting that VSC\#1 is close to the generator and works as Slave-VSC to regulate active power substantially like a constant power load. The swing frequency observed here is about $2 \mathrm{~Hz}$ and typical in the large-signal studies. The transient dynamics of the MTDC grid are dependent on the configurations. For example, the sign of the DC current $i_{\mathrm{dc} \text { line } 12}$ is changed for the green and blue lines (note that no red line appears in the same figure because no line 1-2 exists in this case). Here, we see that the transient dynamics of the DC voltage $v_{\mathrm{dc} 2}$ are almost the same because the voltage is regulated by Master-VSC. The transient dynamics of the other DC voltages $v_{\mathrm{dc} 1}$ and $v_{\mathrm{dc} 3}$ are dependent on the configurations. This is mainly from the fact that the two DC voltages are associated with Slave-VSC for active-power regulation. Therefore, we show that the relation between the configurations and system-wide dynamics are simulated and visualized in the modular modeling. In a case of DC fault, the system-wide dynamics become complicated and possibly multi-scale due to the bidirectional interaction of $\mathrm{AC}$ and MTDC grids. We will show this case in the conference presentation.

\section{CONCLUding REMARKS}

This paper presented the first report on our modular approach to large-signal dynamics of interconnected AC/MTDC grids. The approach wad demonstrated numerically for the four different configurations of the three-terminal DC network. Future works are to improve the control model of VSC and to consider the relevance of the modular modeling in terms of large-signal performance assessment, e.g. by comparison with other modeling frameworks. 

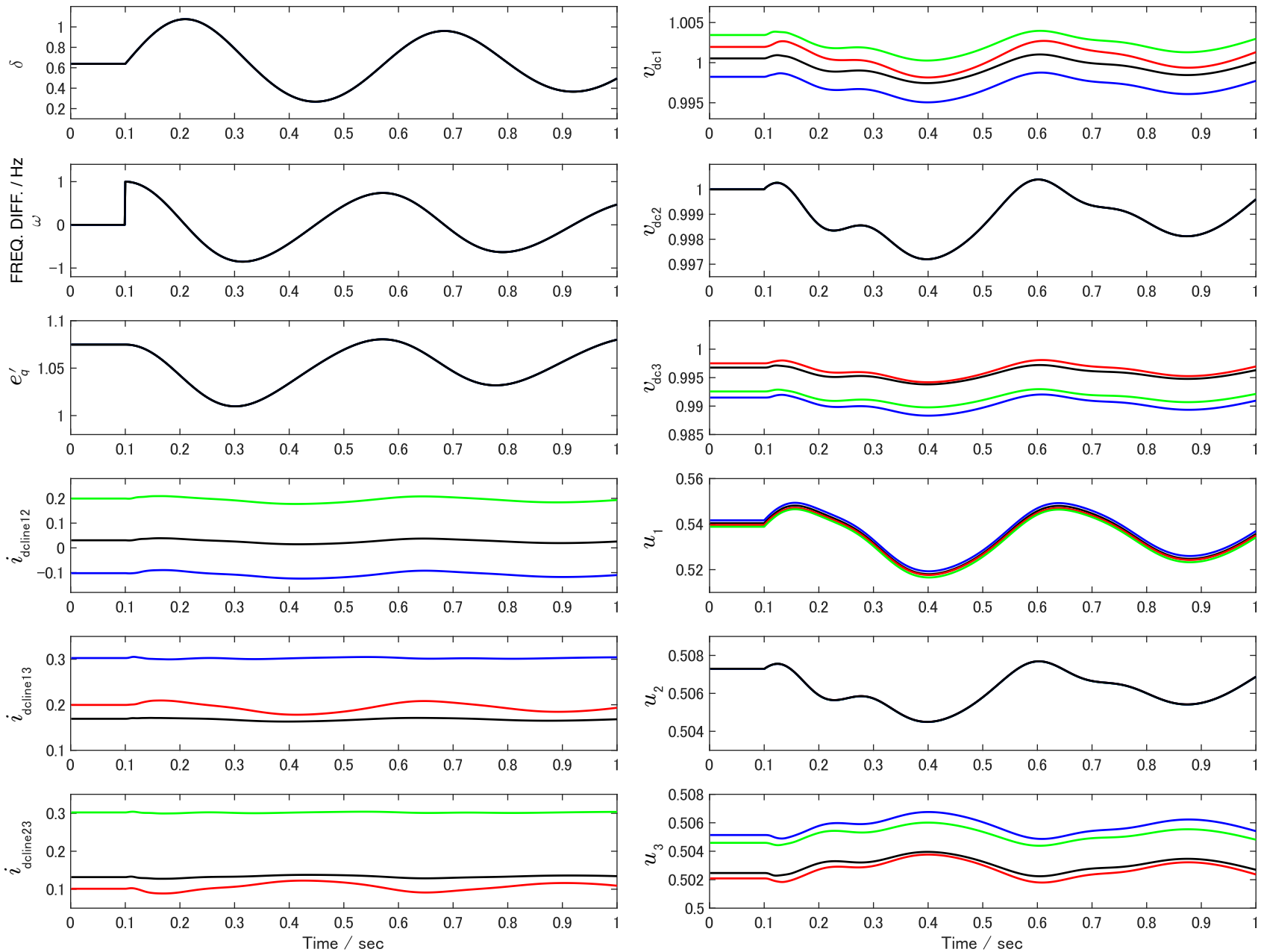

Fig. 5. Numerical simulations of large-signal dynamics in the interconnected system of AC/MTDC grids using the modular modeling: black for the MTDC network in Figure 1 with the three lines (i.e., all-to-all coupling); red for the MTDC network without line 1-2; green for the MTDC network without line 1-3; and blue for the MTDC network without line 2-3.

\section{REFERENCES}

[1] M.K. Bucher, R. Wiget, G. Andersson, and C.M. Franck, Multiterminal HVDC networks-What is the preferred topology?, IEEE Trans. Powe Del., vol.29, no.1, pp.406-413 (2014)

[2] M.A. Pai, K.R. Padiyar, and C. Radhakrishnan, Transient stability analysis of multi-machine AC/DC power systems via energy-function method, of multi-machine AC/DC power systems via energy-function method,
IEEE Trans. Power App. Syst., vol.PAS-100, no.12, pp.5027-5035 (1981)

[3] C.L. DeMarco and C.A. Cañizares, A vector energy function approach for security analysis of AC/DC systems, IEEE Trans. Power Syst., vol.7 no.3, pp.1001-1011 (1992)

[4] Y. Susuki, T. Hikihara, and H.-D. Chiang, Stability boundaries analysis of electric power system with DC transmission based on differentialalgebraic equation system, IEICE Trans. Fund. Electr., vol.E87-A, no.9, pp.2339-2346 (2004)

[5] J. Beerten, S. D'Arco, and J.A. Suul, Cable model order reduction for HVDC systems interoperability analysis, Proc. IET Internationa Conference on AC and DC Power Transmission (2015)

[6] J. Willems, The behavioral approach to open and interconnected systems, IEEE Contr. Syst. Mag., vol.27, issue 6, pp.46-99 (2007)

[7] C. Kojima, Y. Susuki, and S. Hara, Dissipativity-based stability analysis of networked nonlinear descriptor systems and its applications to powe grids, SICE J. Contr. Measure. Syst. Integration, vol.12, no.1, pp.29-38 (2019)

[8] Y. Ohashi, Y. Susuki, A. Ishigame, and T. Funaki, A construction of the dynamic model for interconnected AC/multi-terminal DC transmission systems and its transient simulation, IEE Japan Technical Report \#PSE19-002, January (2019) (in Japanese)

[9] S. Cole, J. Beerten, and R. Belmans, Generalized dynamic VSC MTDC model for power system stability studies. IEEE Transactions on Powe Systems, vol.25, no.3 pp.1655-1662 (2010)
[10] M. Andreasson, R. Wiget, D.V. Dimarogonas, K.H. Johansson, and G. Andersson, Distributed frequency control through MTDC transmission systems, IEEE Trans. Power Syst., vol.32, no.1 pp.250-260 (2017)

[11] A.G. Endegnanew, G. Bergna-Diaz, and K. Uhlen, Avoiding AC/DC grid interaction in MMC based MTDC systems, Proc. 2017 Twelfth International Conference on Ecological Vehicles and Renewable Energies (2017)

[12] D. Jovcic and K. Ahmed, High Voltage Direct-Current Transmission: Converters, Systems and DC Grids, John Wiley \& Sons (2015)

[13] Y. Susuki, Transient Dynamics and Stability Boundaries in Electric Power System with DC Transmission, Ph.D. dissertation, Kyoto University (2005)

[14] H.-D. Chiang, C.-C. Chu, and G. Cauley, Direct stability analysis of electric power systems using energy functions: Theory, applications, and perspective, Proc. IEEE, vol.83, no.11, pp.1497-1529 (1995)

[15] H. Akagi, Y. Kanazawa, and A. Nabae, Instantaneous reactive power compensators comprising switching devices without energy storage components, IEEE. Trans. Ind. Appl., vol.IA-20, no.3, pp.625-630 (1984)

[16] A. Yazdani and R. Iravani, Voltage-Sourced Converters in Power Systems: Modeling, Control, and Applications, John Wiley \& Sons (2010)

[17] X. Li, Z. Yuan, J. Fu, Y. Wang, T. Liu, and Z. Zhu, Nanao multiterminal VSC-HVDC project for integrating large-scale wind generation, Proc. 2014 IEEE PES General Meeting d Conference Exposition (2014)

[18] R. Sandano, M. Farrell, and M. Basu, Enhanced master/slave control strategy enabling grid support services and offshore wind power dispatch in a multi-terminal VSC HVDC transmission system, Renewable Energy, vol.113, pp.1580-1588 (2017)

[19] N.R. Chaudhuri and B. Chaudhuri, Adaptive droop control for effective power sharing in Multi-Terminal DC (MTDC) grids, IEEE Trans. Power Syst., vol.28, no.1 pp.21-29 (2013) 